\title{
A boundary integral approach for determining the pressure error
}

\author{
K. R. Rajagopal and A. Mir-Mohamad-Sadegh
}

With 6 figures

(Received May 21, 1979)

\section{Introduction}

The existence of a systematic difference between the normal stress and the pressure communicated through a hole, due to the flow of a non-Newtonian fluid past the hole, was first inferred by Broadbent et al. (1) and they provided an equation between the pressure error $p_{e}$ and the shearing stress $\tau$ which was empirical. Tanner and Pipkin (2) considered the creeping flow of an incompressible second-order fluid across an infinitely deep slot and they found that the pressure error $p_{e}$ was given through

$p_{e}=-\frac{N_{1}(\kappa)}{4}$,

where $N_{1}(\kappa)$ is the first normal stress difference and $\kappa$ the shear rate. Kearsley (3) found that the pressure error due to the creeping rectilinear flow along an infinitely deep slot of a secondorder fluid was given by

$p_{e}=\frac{N_{2}(\kappa)}{2}$,

where $N_{2}(k)$ is the second normal stress difference. Later, the pressure error due to the rectilinear motion of a second-order fluid between two infinitely parallel plates $D$ apart, the bottom plate with a slot of finite depth $d$ and width $W$, and the top plate moving with a constant speed $V$, was determined by Kearsley (4). He obtained an exact solution to the problem and found that the pressure error $p_{e}$ was given through

$p_{e}=\frac{N_{2}(\kappa)}{2} G(k, \alpha)$,

where $N_{2}(\kappa)$ is evaluated at $\kappa=V / D$ and

$G(k, \alpha)=\frac{1-k^{2}}{1-k^{2} \operatorname{sn}^{2} \alpha}$, with $k$ and $\operatorname{sn} \alpha$ being the modulus and the modular sine respectively. Rajagopal and Huilgol (5) obtained upper and lower bounds to the pressure error for the flow of a second-order fluid in the above domain, where in addition to the top plate moving at a constant speed $V$ they also allowed for a pressure gradient in the flow direction.

While the problem considered by Kearsley (4) leads to solving a Laplace's equation for the velocity, with appropriate boundary conditions, the problem considered by Rajagopal and Huilgol (5) reduces to solving a Poisson's equation for the velocity, with appropriate boundary conditions. The complicated nature of the flow domain, however, makes it exceedingly difficult to solve the problem exactly, and hence we adopt a numerical technique to solve the problem.

In this analysis we devise a boundary integral technique to obtain the pressure error for the problem considered by Rajagopal and Huilgol (5). This technique has the advantage of being accurate, in addition to permitting a more efficient use of the computer in comparison to other numerical techniques. A numerical solution based on this technique for the Laplace's equation, using the classical Green's theorem, was first introduced by Rizzo (6). Later, a direct integral equation for the solution of the Poisson's equation was developed by Jawson and Symm (7). While we could employ a direct boundary integral technique by utilizing the standard Green's identity to solve the problem, we adopt an indirect approach which we feel is better suited for such problems. The indirect approach that we adopt is a modified version of those outlined in Mir-Mohamad-Sadegh and Altiero (8) and Mir-Mohamad-Sadegh (9). 
In section 2 we formulate the problem and obtain an expression for the pressure error. In section 3 after a brief review of the boundary integral technique, in general, we obtain the boundary integral equation corresponding to our problem, and devise a numerical technique for solving the boundary integral equation. We conclude the analysis in section 4 by comparing the results which we obtain with the exact results of Kearsley (4) and the bounds established by Rajagopal and Huilgol (5).

\section{Formulation of the problem}

The Cauchy stress $T$ in an incompressible homogeneous fluid of second-order is assumed to be related to the fluid motion in the following manner (10):

$T=-p \mathbf{1}+\mu A_{1}+\beta A_{1}^{2}+\gamma A_{2}$,

where $\mu$ is the viscosity, $\beta$ and $\gamma$ the normal stress moduli, $-p 1$ the constitutively indeterminate spherical stress due to the constraint of incompressibility. $A_{1}$ and $A_{2}$ are the first two RivlinEricksen tensors given through

$A_{1}=\operatorname{grad} \boldsymbol{v}+(\operatorname{grad} \boldsymbol{v})^{T}$,

and

$A_{2}=\dot{A}_{1}+A_{1}(\operatorname{grad} v)+(\operatorname{grad} v)^{T} A_{1}$.

The dot in eq. $[2.2]_{2}$ denotes material time differentiation.

Since the fluid obeys the balance of linear momentum, we obtain that

$\operatorname{div} \boldsymbol{T}+\rho \boldsymbol{b}=\rho \dot{\boldsymbol{v}}$,

where $\boldsymbol{b}$ is the specific body force. The constraint of incompressibility permits the fluid to undergo only isochoric motions, and hence

$\operatorname{div} \boldsymbol{v}=0$.

We are interested in the determination of the velocity field in a cartesian co-ordinate system corresponding to the rectilinear flow of a fluid, whose stress in characterized by eq. [2.1], along the $z$-axis in a domain as shown in figure 1 , such that

$\dot{x}=0, \quad \dot{y}=0 \quad$ and $\quad \dot{z}=w(x, y)$.

\footnotetext{
1) The constitutive relation [2.1] has been shown to be a second-order approximation in the sense of retardations to all simple fluid models (10). However, the relation [2.1] has also been considered as an exact model for some fluid in its own right $(11,12)$.
}

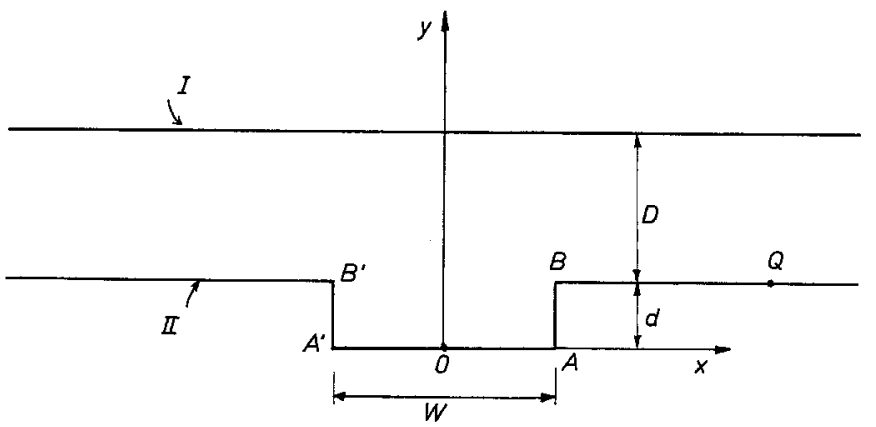

Fig. 1. The flow domain

As has been shown in (5), if one assumes that the body forces can be neglected, one can show that in a cartesian co-ordinate system, eqs. [2.3] and [2.4] for the creeping rectilinear steady flow of an incompressible second-order fluid yield

$\Delta w=\frac{1}{\mu} \frac{\partial p}{\partial z}=C$,

where $C$ is a non-positive constant, $w=w(x, y)$ the velocity in the $z$-direction and $\Delta$ is the twodimensional Laplacian operator

$\Delta \equiv \frac{\partial^{2}}{\partial x^{2}}+\frac{\partial^{2}}{\partial y^{2}}$.

The velocity field $w$ satisfies the following boundary conditions (see fig. 1)

$w= \begin{cases}V & \text { on } \quad y=d+D, \\ 0, & |x| \leq \frac{W}{2}, \quad y=0, \\ 0, & |x|=\frac{W}{2}, \quad 0 \leq y \leq d, \\ 0, & |x| \geq \frac{W}{2}, \quad y=d,\end{cases}$

$w \rightarrow w(y)$ as $|x| \rightarrow \infty$.

Kearsley (4) has shown that if $\left(w, p_{0}\right)$ is a Newtonian velocity pressure pair that satisfies [2.5], then the velocity pressure pair $(w, p)$ corresponding to a second-order fluid satisfies [2.5] if

$p=p_{0}-C(2 \gamma+\beta) w$.

Thus using eqs. [2.1] and [2.9], one can calculate the normal stress $T_{y y}$ to be

$T_{y y}=-p^{0}+\frac{1}{2}(2 \gamma+\beta)\left[\left(w_{, x}^{2}-w_{, y}^{2}\right)-2 C w\right]$,

where comma denotes partial differentiation and $w_{, x}^{2}=(\partial w / \partial x)^{2}$, etc. 
The pressure error made in measuring the pressure at 0 (see fig. 1) would then be the difference between the undisturbed normal stress at $\pm \infty$ and the normal stress at 0 . Hence,

$p_{e}=T_{y y}( \pm \infty, d)-T_{y y}(0,0)$.

On the bottom plate with the slot, both $w$ and $w_{, x}$ are zero. Therefore, by virtue of eq. [2.10], eq. [2.11] can be rewritten as

$p_{e}=\frac{2 \gamma+\beta}{2}\left[w_{, y}^{2}( \pm \infty, d)-w_{, y}^{2}(0,0)\right]$.

Eq. [2.12] can be further simplified into the form

$p_{e}=G \frac{\bar{N}_{2}}{2}$,

where

$G \equiv\left[1-\frac{w_{, y}^{2}(0,0)}{w_{, y}^{2}( \pm \infty, d)}\right]$,

$\bar{N}_{2}$ is the normal stress difference based on the shear rate at $x= \pm \infty$ and $y=d$.

Thus, the determination of the pressure error reduces to the determination of the function $G$. The function $G$ can be determined by obtaining the velocity field $w$ which satisfies eqs. [2.6] and [2.7]. In the next section we devise a numerical technique for obtaining $w_{, y}$ and hence $G$.

\section{Numerical technique}

\section{a) Formulation of the boundary integral}

Let $w$ be the solution to the partial differential equation

$\Delta w=\phi(x, y, z)$ in $D$,

subject to the boundary conditions

$w=f(x, y, z)$ on $\partial D_{1}$,

$\frac{\partial w}{\partial n}=g(x, y, z)$ on $\quad \partial D_{2}$,

where $\partial D=\partial D_{1} \bigcup \partial D_{2}$ is the boundary of the domain $D$ and $n$ is the co-ordinate in the direction of the outward unit normal to the boundary $\partial D$. Suppose, we embed the domain $D$ in an infinite space $D^{\prime}$ and subject the boundary $\partial D$ of $D$ to a layer of source points $P^{*}(x, y, z)$ where $(x, y, z) \in \partial D$, and define a function $\bar{w}$ through

$\bar{w}(\boldsymbol{x})=w(\boldsymbol{x})-\int_{D} \phi(\boldsymbol{\xi}) G(\boldsymbol{x}, \boldsymbol{\xi}) d v(\boldsymbol{\xi}) ;$ we use the notation $x=(x, y, z)$ and $\xi=(\xi, \eta, \zeta)$, $d v(\xi)$ denotes the volume measure in three dimensions and $G(\boldsymbol{x}, \boldsymbol{\xi})$ denotes the Green's function which is the fundamental solution ${ }^{2}$ ) to the Laplace's equation. Then, by virtue of eq. [3a.1], we obtain that

$\Delta \bar{w}=0$ in $D$,

and hence, by virtue of the superposition principle, $\bar{w}$ can be represented as

$\bar{w}(\boldsymbol{x})=\int_{\partial D} P^{*}(\xi) G(\boldsymbol{x}, \xi) d a(\xi)$,

where $d a(\xi)$ denotes the usual area measure. On substituting eq. [3a.5] in eq. [3a.3], we obtain that

$$
\begin{aligned}
w(\boldsymbol{x})= & \int_{\partial D} P^{*}(\boldsymbol{\xi}) G(\boldsymbol{x}, \boldsymbol{\xi}) d a(\xi) \\
& +\int_{D} \phi(\xi) G(\boldsymbol{x}, \boldsymbol{\xi}) d v(\xi) .
\end{aligned}
$$

The layer of source points $P^{*}(\xi)$ is unknown in the above equation and can be obtained by employing the boundary conditions [3a.2]. Thus, when $\boldsymbol{x}^{\prime} \in \partial D_{1}$,

$$
\begin{aligned}
& \int_{\partial D} P^{*}(\xi) G(\boldsymbol{x}, \boldsymbol{\xi}) d a(\boldsymbol{\xi}) \\
& \quad=f\left(\boldsymbol{x}^{\prime}\right)-\int_{D} \phi(\boldsymbol{\xi}) G\left(\boldsymbol{x}^{\prime}, \boldsymbol{\xi}\right) d v(\boldsymbol{\xi}),
\end{aligned}
$$

and, when $\boldsymbol{x}^{\prime} \in \partial D_{2}$,

$$
\begin{aligned}
\int_{\partial D} P^{*}(\xi) \frac{\partial}{\partial n_{x^{\prime}}} G\left(\boldsymbol{x}^{\prime}, \boldsymbol{\xi}\right) d a(\boldsymbol{\xi}) \\
\quad=g\left(\boldsymbol{x}^{\prime}\right)-\int_{D} \phi(\boldsymbol{\xi}) \frac{\partial}{\partial n_{x^{\prime}}} G\left(\boldsymbol{x}^{\prime}, \boldsymbol{\xi}\right) d v(\boldsymbol{\xi}) .
\end{aligned}
$$

In the above eq. [ $3 \mathrm{a} .8$ ] $\partial / \partial n_{x^{\prime}}$ denotes the derivative in the direction of the outward normal at the point $\boldsymbol{x}^{\prime}$. We note that the integral on the left-hand side of eq. [3a.8] is a singular integral and its value can be found to be $\frac{1}{2} P^{*}\left(x^{\prime}\right),(14)$. The indirect integral eqs. [3a.7] and [3a.8] can be solved for $P^{*}(\xi)$, and once $P^{*}(\xi)$ is obtained, we can determine $w(\boldsymbol{x})$ through eq. [3a.6]. In the next subsection we outline a numerical scheme for solving eqs. [3a.7] and [3a.8].

$\left.{ }^{2}\right)$ The fundamental solution, cf. (13), to the Laplace's equation in three (two) dimensional domains is

$G(\boldsymbol{x}, \boldsymbol{\xi})=\frac{1}{4 \pi r}, \quad\left(G(\boldsymbol{x}, \boldsymbol{\xi})=\frac{1}{2 \pi} \log r\right)$,

where $r$ denotes the distance of point $(x, y, z)((x, y))$ from the point $(\xi, \eta, \zeta)((\xi, \eta))$. 


\section{b) Numerical solution}

On subdividing the boundary $\partial D$ into $N$ discrete intervals $\Delta S_{i}(i=1, \ldots, N)$, and assuming that $P^{*}\left(x^{\prime}\right), f\left(x^{\prime}\right)$ and $g\left(x^{\prime}\right)$ are constant over each of the intervals, we can rewrite eqs. $[3 \mathrm{a} .7]$ and $[3 \mathrm{a} .8]$ in the form

$$
\begin{aligned}
& \sum_{j=1}^{N}\left[\int_{\Delta S_{j}} G(i, j) d a_{j}\right] P_{j}^{*} \\
& =f_{i}-\int_{D} \phi_{k} G(i, k) d v_{k},
\end{aligned}
$$

and

$$
\begin{gathered}
\frac{P_{i}^{*}}{2}+\sum_{\substack{j=1 \\
j \neq i}}^{N}\left[\int_{\Delta S_{j}} \frac{\partial}{\partial n_{i}} G(i, j) d a_{j}\right] P_{j}^{*} \\
=g_{i}-\int_{D} \phi_{k} \frac{\partial}{\partial n_{i}} G(i, k) d v_{k} .
\end{gathered}
$$

In the above equations, $i$ and $j$ as a subscript represent the value at the mid point of the intervals and $i$ and $j$ also denote the co-ordinates of the variables $\boldsymbol{x}^{\prime}$ and $\xi$, respectively.

The eqs. [3b.1] and [3b.2] can be written in the following matrix form:

$\left[K_{i j}\right]\left\{P_{j}^{*}\right\}=\left\{H_{i}\right\}-\left\{E_{i}\right\}$,

where $\left[K_{i j}\right]$ is a $N \times N$ matrix whose elements contain boundary integrals, $\left\{P_{j}^{*}\right\}$ is a $N \times 1$ vector whose elements contain the unknown source points, $\left\{H_{i}\right\}$ is a $N \times 1$ vector whose elements contain the boundary values and $\left\{E_{i}\right\}$ is a $N \times 1$ vector whose elements contain the integral over the domain $D$. Once eq. [3b.3] has been solved for the vector $P_{j}^{*}$, the solution at any point in $D$ can be found from

$w(\boldsymbol{x})=\sum_{j=1}^{N}\left[\int_{\Delta S_{j}} G(\boldsymbol{x}, j) d a_{j}\right] P_{j}^{*}+\int_{D} \phi_{k} G(\boldsymbol{x}, k) d v_{k}$.

\section{c) Solution for the slot}

We shall now consider the problem of the flow of a second-order fluid in the domain shown in figure 1. For this problem, the equations of motion reduce to form

$\Delta w=\phi(x)$ in $D$,

and the boundary conditions to the form

$w=f(x)$ on $\partial D$.

The fundamental solution $G(x, \xi)$ for the twodimensional problem reduces to
$G(x, \xi)=\frac{1}{2 \pi} \log r$,

and hence, on substituting [3c.3] into eq. [ 3 a.7] we obtain that

$$
\begin{aligned}
& \frac{1}{2 \pi} \int_{\partial D} P^{*}(\xi) \log r\left(x^{\prime}, \boldsymbol{\xi}\right) d a(\xi) \\
& \quad=f\left(x^{\prime}\right)-\frac{1}{2 \pi} \int_{D} \phi(\xi) \log r\left(\boldsymbol{x}^{\prime}, \boldsymbol{\xi}\right) d v(\boldsymbol{\xi}) .
\end{aligned}
$$

As $\boldsymbol{\xi}$ approaches $\boldsymbol{x}^{\prime}$ the integral on the left-hand side of eq. [3c.4] becomes singular. However, we avoid this singularity by making the assumption that $P^{*}(\xi)$ is constant over the interval $\Delta S$ and integrate over the path shown in figure 2 . Thus, eq. [3c.4] can be re-written as

$$
\begin{aligned}
\frac{P_{\Delta S}^{*}}{2 \pi} & \Delta S \log \left(\left|\frac{\Delta S}{2}\right|-1\right) \\
& +\frac{1}{2 \pi} \int_{\partial D-\Delta S} \log r\left(\boldsymbol{x}^{\prime}, \xi\right) P^{*}(\xi) d a(\xi) \\
= & f\left(\boldsymbol{x}^{\prime}\right)-\Phi\left(\boldsymbol{x}^{\prime}\right)
\end{aligned}
$$

where

$$
\Phi\left(\boldsymbol{x}^{\prime}\right) \equiv \frac{1}{2 \pi} \int_{D} \phi(\boldsymbol{\eta}) \log r\left(\boldsymbol{x}^{\prime}, \boldsymbol{\eta}\right) d a(\boldsymbol{\eta})
$$

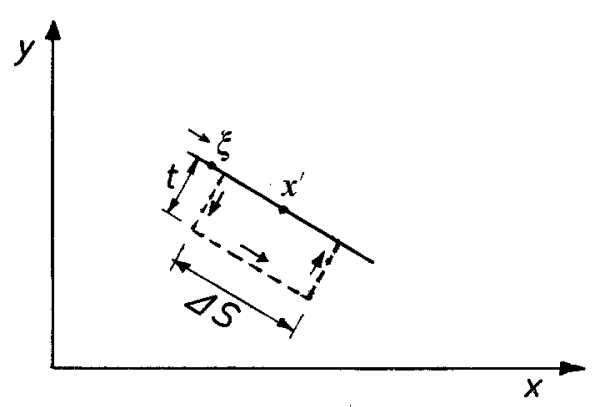

Fig. 2. The integration contour

Eq. [3c.5] can be further rewritten in the form

$$
\begin{aligned}
& \frac{P_{i}^{*}}{2 \pi} \Delta S_{i}\left(\log \left|\frac{\Delta S_{i}}{2}\right|-1\right) \\
& \quad+\frac{1}{2 \pi} \sum_{\substack{j=1 \\
j \neq i}}^{N} P_{j}^{*} \log r_{i j} \Delta S_{j}=f_{i}-\Phi_{i},
\end{aligned}
$$

where $i$ and $j$ are defined in section $3 \mathrm{a}$. Defining the resultant source point at the midpoint of each interval through

$\mathbb{P}_{k}^{*}=\int_{\Delta S_{k}} P_{k}^{*} d a_{k}$ 
and using the trapezoidal rule, eq. [3c.7] can be put into the form

$$
\begin{aligned}
& {\left[\log \left|\frac{\Delta S_{i}}{2}\right|-1+\sum_{\substack{j=1 \\
j \neq i}}^{N} \log r_{i j}\right]\left\{\mathbb{P}_{j}^{*}\right\}} \\
& \quad=2 \pi\left[\left\{f_{i}\right\}-\left\{\Phi_{i}\right\}\right],
\end{aligned}
$$

which we can recognize to be of the form of eq. [3b.3]. Thus, solving eq. [3c.9] for $\mathbb{P}_{j}^{*}$ and substituting this into eq. $[3 \mathrm{~b} .4]$, we can obtain $w(\boldsymbol{x})$. However, since the pressure error is only a function of $\partial w / \partial y$, we are only interested in obtaining

$$
\begin{aligned}
\frac{\partial w}{\partial y}= & \frac{1}{2 \pi} \sum_{j=1}^{N} \frac{y-\eta_{j}}{\left(x-\xi_{j}\right)^{2}+\left(y-\eta_{j}\right)^{2}} \mathbb{P}_{j}^{*} \\
& +\frac{1}{2 \pi} \int_{D} \frac{\phi(x, y)(y-\eta)}{(x-\xi)^{2}+(y-\eta)^{2}} d a .
\end{aligned}
$$

In our problem, since $\phi(x)=C$ and since $f(x, y)$ is either a constant $V$ or zero, the integral [3c.6] and the integral in eq. [3c.10] can be calculated easily, and hence $\partial w / \partial y$ determined.

\section{d) Computation}

The boundary is subdivided into 90 meshes as shown in figure 3 . Using the non-dimensional quantity

$T \equiv \frac{C D^{2}}{V}$

a computer program has been written to calculate $\mathbb{P}^{*}$ from eq. [3c.9]. Choosing the two field points as shown in figure 3 , the value of $\partial w / \partial y$ at the two points has been calculated. The function $G$ which appears in the pressure error eq. [2.13] is then computed. The results have been plotted as graphs in figures 4 to 6 .

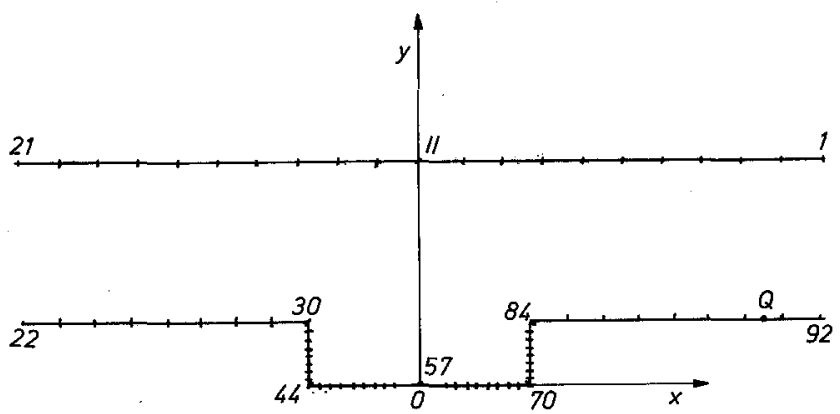

Fig. 3. The flow domain with the meshes used in the numerical computation

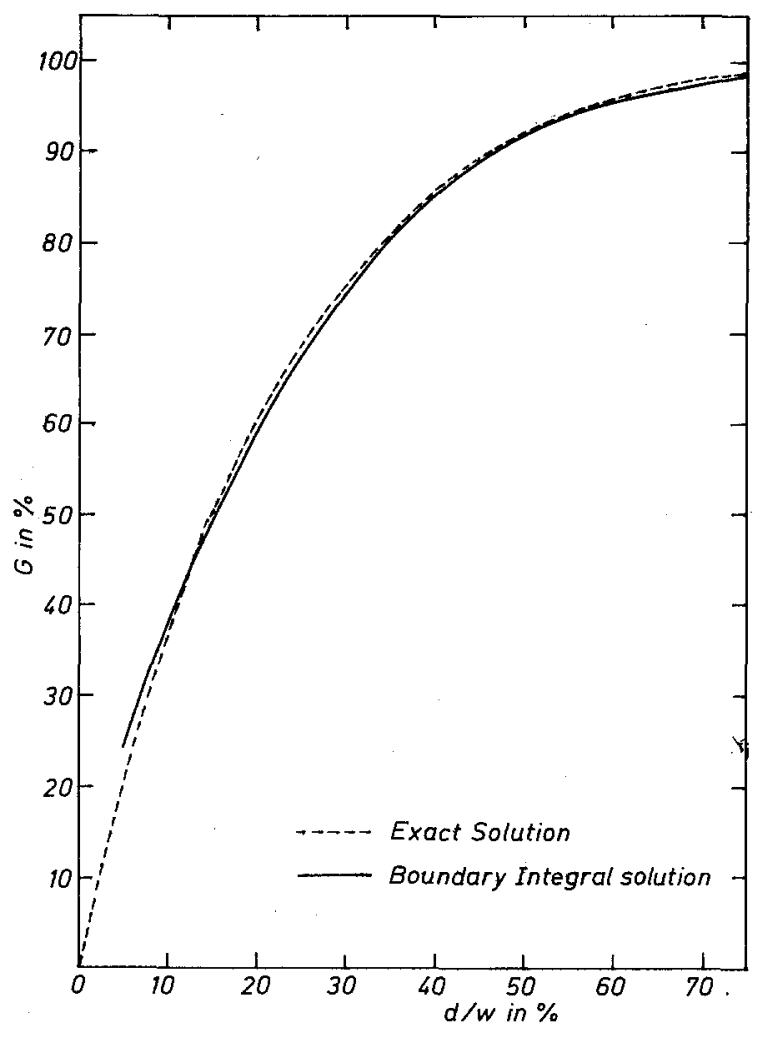

Fig. 4. The boundary integral equation results compared with the exact solution for $T=0$ and $D / W=1$

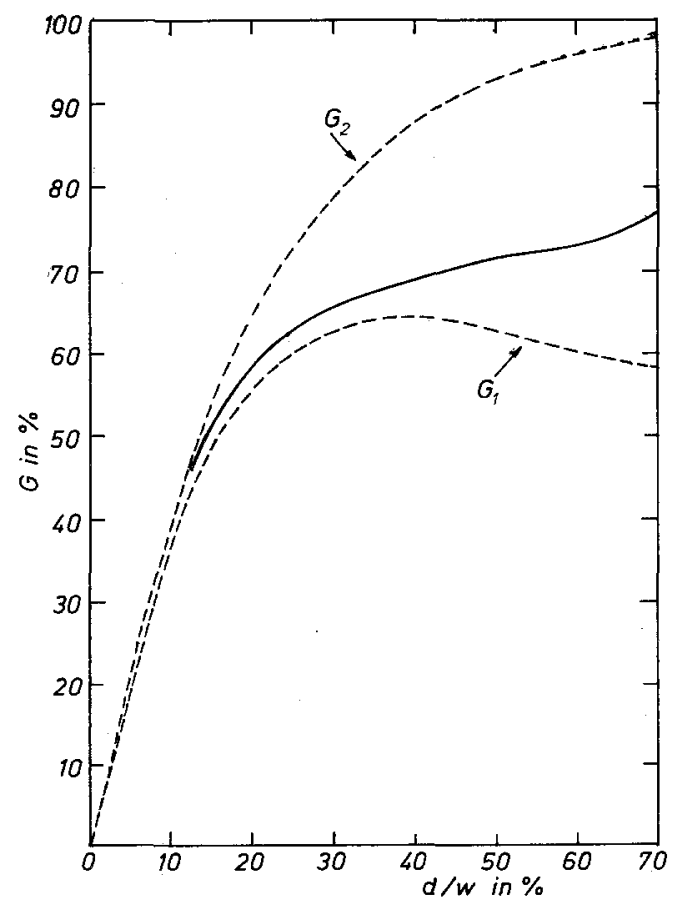

Fig. 5. Upper and lower bounds $G_{1}$ and $G_{2}$ and the B.I.E. solution when $c \neq 0, V>0, T=0.25, D / W=0.5$ 


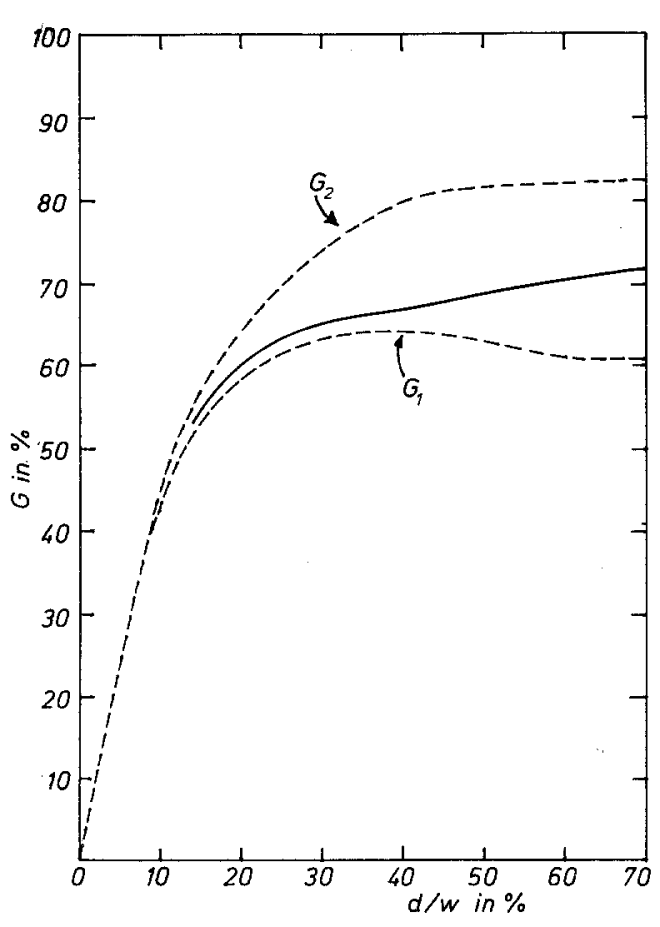

Fig. 6. Upper and lower bounds $G_{1}$ and $G_{2}$ and the B.I.E. solution when $c \neq 0, V>0, T=0.5, D / W=0.5$

\section{Results and discussion}

When the non-dimensional parameter $T=0$, which corresponds to the problem considered by Kearsley (4), we find that our numerical results compare very favorably with the exact solution obtained by Kearsley, as shown in figure 4 . When $T \neq 0$; i.e. when there are pressure gradients along the direction of the flow, we find that our numerical solution lies within the bounds established by Rajagopal and Huilgol (5), as shown in figures 5 and 6 . We have plotted graphs of $G$ vs. $d / W$ for a fixed value of $D / W$ for $T=0.25$ and $T=0.5$. One can easily observe from figures 5 and 6 that when $d / W$ is small in the sense that $d / W$ is less than $50 \%$, the numerical solution is closer to the lower bound, while for large $d / W$ the numerical solution is closer to the upper bound. It can be observed in all the cases considered, the function $G$ peaks rather fast.

For very small values of $d / W$, namely when $d / W$ is less than $10 \%$, our numerical scheme does not provide very good results. This is because when $d / W$ is very small, the corners $A$ and $B\left(A^{\prime}\right.$ and $\left.B^{\prime}\right)$ approach each other (see fig. 1) and this creates problems in the computation since the two singularities due to the two corners coalesce. This problem could possibly be overcome by smoothing out the corners, cf. (7). However, since the upper and lower bounds obtained by Rajagopal and Huilgol (5) are very close to each other (see figs. 4 to 6 ) in this range for $d / W$, we restricted our attention to computing $G$ for values $d / W>10 \%$. Also to ensure better accuracy in our computation, we allowed the intervals containing our field points $O$ and $Q$ to be longer than the other intervals so as to reduce the effect due to the source points in neighboring intervals.

\section{Summary}

The rectilinear flow of a second-order fluid is considered between two infinitely wide and long parallel plates. The bottom plate is at rest and has a slot of depth ' $d$ ' and width ' $W$ ' while the top plate is flat and moves along the flow direction with constant speed. A boundary integral equation technique is developed to determine the pressure error due to such a flow. We find that our numerical results compare favorably with the exact solutions obtained by Kearsley for a special case. Also our results lie within the analytical bounds established by Rajagopal and Huilgol for the pressure error for such flows.

\section{Zusammenfassung}

Es wird die geradlinige Strömung einer Flüssigkeit zweiter Ordnung zwischen zwei unendlich ausgedehnten parallelen Platten betrachtet. Die Bodenplatte ruht und ist mit einer rechteckigen Nute der Tiefe $d$ und der Breite $W$ versehen, wohingegen die flache Deckplatte mit konstanter Geschwindigkeit in Strömungsrichtung bewegt wird. Eine Integralgleichungsmethode (boundary integral equation technique) wird entwickelt zur Bestimmung des durch die Nute in einer derartigen Strömung hervorgerufenen Druck-Fehlers (pressure error). Es wird gefunden, daß die auf diese Weise erhaltenen numerischen Ergebnisse gut mit den für einen Spezialfall von Kearsley berechneten exakten Lösungen iibereinstimmen. Gleichfalls liegen die Ergebnisse innerhalb der von Rajagopal und Huilgol für solche Strömungen auf analytische Weise erhaltenen Grenzkurven.

\section{References}

1) Broadbent, J. M., A. Kaye, A. S. Lodge, D. G. Vale, Nature 217, 55 (1968).

2) Tanner, R. I., A. C. Pipkin. Trans. Soc. Rheology 13, 471 (1969). (1970)

3) Kearsley, E. A., Trans. Soc. Rheology 14, 419

4) Kearsley, E. A., Lecture at the 44th Annual Meeting of Soc. of Rheology (Montreal 1973).

5) Rajagopal, K. R., R. R. Huilgol, Rheol. Acta 18, 456 (1979).

6) Rizzo, F. J., Boundary-Integral Equation Method: Computational Applications in Applied Mechanics, AMD, ASME (1975). 
7) Jawson, M.A., G. J. Symm, Integral Equation Methods in Potential Theory and Elastostatics, Academic Press (London-New York-San Francisco 1977).

8) Mir-Mohamad-Sadegh, A., N. J. Altiero, Intl. J. Eng. Fracture Mechanics 11, 831 (1979).

9) Mir-Mohamad-Sadegh, A., Ph. D. Thesis, Michigan State University (1978).

10) Coleman, B. D., W. Noll, Arch. Ratl. Mech. Anal. 6, 355 (1974).

11) Dunn, J. E., R. L. Fosdick, Arch. Ratl. Mech. Anal. 56, 191 (1974)

12) Fosdick, R. L., K. R. Rajagopal, Intl. J. NonLinear Mechanics 13, 131 (1978).
13) Courant, R., D. Hilbert, Methods of Mathematical Physics, Vol. 1, Interscience (New York 1970).

14) Cruse, T. A., Mathematical Foundations of the Boundary-Integral Equation Methods in Solid Mechanics, AFOSR-TR-77-1002 (1977).

Authors' address:

Dr. K. R. Rajagopal, Dr. A. Mir-Mohamad-Sadegh

Department of Applied Mechanics and

Engineering Science

The University of Michigan

Ann Arbor, Michigan 48109 (U.S.A) 